\title{
Von den Flitterwochen zur distanzierten Partnerschaft
}

\section{Deutsche Manager in internationalen M\&A}

\author{
Martina Fuchs $^{1} \cdot$ Martin Schalljo $^{1}$
}

Online publiziert: 28. Februar 2017

(c) Der/die Autor(en) 2017. Dieser Artikel ist eine Open-Access-Publikation.

\begin{abstract}
Zusammenfassung Grenzüberschreitende Mergers \& Acquisitions (M\&A) stellen große interkulturelle Anforderungen an die beteiligten Führungskräfte. Derzeit fordern die Übernahmewellen einerseits von Private-Equity Investoren und andererseits von Investoren aus den Emerging Economies die deutschen Manager heraus. Wenn es sich - wie in dieser Untersuchung - um freundliche Übernahmen handelt, begrüßen die Führungskräfte zunächst die neuen Eigner. Die hier verfolgte Analyse strukturaler Hermeneutik entdeckt allerdings latente Tiefenstrukturen einer normativen Professionsethik in den Interviews, die zu Praktiken der Distanzierung führen, und zwar besonders gegenüber den jungen Investoren aus China, Indien und Russland.
\end{abstract}

\begin{abstract}
Cross-border mergers and acquisitions (M\&A) require considerable intercultural openness on the part of the managers involved. A recent wave of takeovers by private equity investors and foreign investors from emerging economies has challenged German executives. In cases of friendly takeovers, as described in this study, managers at first appear to extend a cosmopolitan welcome to the new owners. However, further analysis of structural hermeneutics reveals latent normative professional ethics of managers which lead to practices of distancing, in particular from young investors from China, India, and Russia.
\end{abstract}

Prof. Dr. Martina Fuchs

fuchs@wiso.uni-koeln.de

Dipl.-Geogr. Martin Schalljo

schalljo@wiso.uni-koeln.de

1 Wirtschafts- und Sozialgeographisches Institut, Wirtschaftsund Sozialwissenschaftliche Fakultät, Universität zu Köln, Albertus-Magnus-Platz, 50923 Köln, Deutschland

\section{Einleitung}

Viele Geographeninnen und Geographen arbeiten an Brückenköpfen, die verschiedene internationale Organisationen miteinander verbinden. Für ihre Berufspraxis ist es wichtig zu verstehen, wie latente sozio-kulturelle Deutungsmuster zu Annäherung bzw. Distanzierung führen und wie sozio-kulturelle Differenzen zu überwinden sind, da diese Prozesse über strategische raumbezogene Entscheidungen in globalen Zusammenhängen bestimmen.

Ein laufendes Forschungsvorhaben über „Deutungsmuster von Führungskräften bei Übernahmen und Beteiligungen durch ausländische Investoren" untersucht soziokulturelle Deutungsmuster deutscher Manager am Beispiel grenzüberschreitender Merger \& Acquisitions (M\&A). Solche Unternehmensübernahmen bilden für Führungskräfte oft kritische Ereignisse. Die Manager nehmen wahr, dass ihre ausländischen Geschäftspartner nach anderen Regeln als sie selbst spielen. Schnell ist dann von ,interkulturellen Unterschieden" die Rede. Eine Reflexion der normativen sozio-kulturellen Deutungsmuster, die solchen Zuschreibungen von Anderssein zugrunde liegen, bleibt dabei aus. Ein fehlendes „Wir“ beeinträchtigt zwar den Post Merger Prozess nicht zwangsläufig, ist aber oft für den längerfristigen Unternehmenserfolg problematisch (Rottig et al. 2013). Insofern wird der Post Merger Prozess in der Managementliteratur als harte Beziehungsarbeit nach den Flitterwochen der jungen Akquisition beschrieben (Cartwright und Cooper 2001).

Dieser Beitrag arbeitet aus Interviews mit Managern über ihre Erfahrungen in M\&A die darunterliegende normative Grammatik von Nähe und Distanz heraus. Solche Tiefenstrukturen (oder bedeutungsstrukturierende „frames“, Goffman 1974) sind den Führungskräften selbst weitgehend unbewusst, da sie diese Muster in Ausbildung, Studium und 
ihrer Erfahrungswelt als Manager internalisiert haben. Diese latente Professionsethik prägt wesentlich das Handeln der Manager, da sie je nach Situation mobilisiert wird und ihr Handeln lenkt. Die Frage lautet: Welche professionsethischen Tiefenstrukturen lenken die Praktiken deutscher Manager, wenn ihre Unternehmen von ausländischen Investoren übernommen worden sind?

Der Beitrag bezieht sich dabei auf zwei junge Wellen von M\&A. Seit den 1990er-Jahren gibt es zahlreiche internationale Übernahmen durch westliche Private-Equity Investoren. Mittlerweile ist die Metapher von „Heuschrecken“ dem Bild von wichtigen Finanzgebern gewichen (Brettel et al. 2008, S. 8). Aktuell gibt es eine weitere Übernahmewelle durch Investoren aus jungen Wachstumsökonomien, den sogenannten Emerging Economies. In den Medien werden diese Investoren als finanzstarke und langfristig orientierte Anleger bei ihrer Ankunft überwiegend begrüßt (Golinski und Henn 2015). Aber unbeantwortet ist bislang die Frage, wie sich die Post Merger Prozesse im weiteren Verlauf gestalten.

Der Beitrag erläutert im Folgenden die Methode und erklärt dann das Konzept der Professionsethik. Die Ergebnisse zeigen auf, wie bei allen Investoren in der frühen Post Merger Phase zunächst Deutungsmuster von kosmopolitischer Offenheit und Lernen als Grundprinzip mobilisiert werden. Während sich dann bei Managern, die von westlichen Private Equity-Investoren übernommen worden sind, vor allem ein Anpassungspragmatismus zeigt, da die Diskrepanzen zwischen eigener Professionsethik und dem wahrgenommenen Verhalten des ausländischen Gegenübers eher gering sind, zeigen sich gegenüber Investoren aus Emerging Economies Friktionen zwischen dem, was deutsche Manager als Elemente ihrer Professionsethik verinnerlicht haben (wie legitime Hierarchien, funktionale Administration und professionelle Expertise) und dem, was sie bei den internationalen Gegenübern wahrnehmen. Distanzierung ist in dem Fall das Ergebnis.

\section{Methode}

2014/2015 wurden 17 Führungskräfte in 15 deutschen mittelständischen Unternehmen interviewt, die zuvor vollständig von ausländischen Investoren übernommen worden waren. Allen Gesprächspartnern ist gemeinsam, dass sie als „westliche“ bzw. deutsche Manager sozialisiert sind. Sie weisen internationale Erfahrung auf, sind aber in Deutschland geboren bzw. leben seit langem hier und sind größtenteils durch frühere Arbeit in deutschen Traditionsunternehmen geprägt. Mittels strukturaler Hermeneutik wurden in den Interviewprotokollen die grundlegenden sozio-kulturellen Regeln analysiert, die als Sprachmuster handlungswirksam werden und damit eine soziale Praxis konstituie- ren. Diese Regeln sind versteckte Alltagstheorien, die erst in kritischen Situationen in Erscheinung treten (Oevermann 1973/2001a, 2001b). Die Methode der strukturalen Hermeneutik ist überaus aufwändig und kann in diesem Beitrag nicht im Einzelnen erklärt werden (vgl. hierzu Fuchs und Schalljo 2016). Im Prinzip beruht sie darauf, dass das Interviewprotokoll als Sequenz von Handlungen verstanden wird. Die relevanten Äußerungen werden auf ihren Sinngehalt hin geprüft, besonders indem sie evidenzbasiert durch mögliche andere Formulierungen ersetzt werden. Das Ziel ist die Entdeckung der zugrunde liegenden Logik der Professionsethik als sozio-kulturell verankertes Sinnkonstrukt. Für die strukturale Hermeneutik ist die Zahl der hier analysierten 17 Interviews hoch, da die Methode einzelfallbezogen arbeitet; der Gewinn dieser qualitativen Analyse liegt darin, milieuspezifische Deutungen und Handlungslogiken aufzudecken.

\section{Konzeptionelle Annäherung: Professionsethik}

Max Weber (1922/1985, S. 35-37) beschrieb die Prinzipien der modernen Organisation mit legitimer Herrschaft und funktionaler Administration; Joseph Schumpeter (1928) steht für die Idee von Führungskräften als verantwortliche Unternehmer mit hoher Handlungsautonomie. Diese Maximen prägen bis heute die Professionsethik von Führungskräften. Zudem ist professionelle Expertise entscheidend; eigene Kompetenzen werden weitergegeben, wie auch selbst neuen Herausforderungen lernend begegnet wird (Bathelt und Gertler 2005). Im Zeitalter der Globalisierung umfasst die Professionsethik zudem besonders, sich selbst als kosmopolitisch offene Persönlichkeiten zu sehen und weitgehend vorurteilsfrei Menschen aus fernen Ländern zu begegnen. Heutzutage wird die Professionsethik von deutschen - bzw. im weiteren Sinne, westlichen ' - Führungskräften durch Managementschulen und Universitäten weiter konkretisiert, indem detaillierte Leistungskennzahlen und Modell-Kalkulationen die Prozesse überprüfbar machen (Bitterer und Heeg 2015, S. 38).

Die Professionsethik bildet den latenten Prüfstein, wie Manager die jeweilige Situation im M\&A bewerten. Treten Friktionen zwischen, eigentlich ' vorhandener Norm und dem wahrgenommenen Verhalten des internationalen Geschäftspartners auf, kann das von Seiten der Manager mit pragmatischer Anpassung an die Gegebenheiten beantwortet werden, oder aber mit dem Versuch, durch Distanzierung die eigene Handlungsautonomie zu wahren (Abb. 1). 


\begin{tabular}{|c|}
\hline WESTLICHE PROFESSIONSETHIK: \\
Verantwortlichkeit für das Unternehmen \\
Legitime Hierarchien \\
Funktionale Administration \\
Fachliche Expertise und Lernen als Grundprinzip \\
Kosmopolitische Offenheit \\
Passung oder Diskrepanz zum wahrgenommenen \\
Verhalten des internationalen Geschäftspartners \\
aus den Emerging Economies: \\
ANPASSUNGSPRAGMATISMUS $\leftrightarrow$ DISTANZIERUNG \\
\hline
\end{tabular}

Abb. 1 Professionsethik von Führungskräften, eigene Abbildung

\section{Ergebnisse: Professionsethik setzt Maßstäbe für die Positionierung der deutschen Manager gegenüber den ausländischen Investoren}

Alle befragten Manager betonten anfänglich und nachdrücklich im Gespräch, dass es sich in ihren Fällen um freundliche Akquisitionen gehandelt habe und dass sie zumindest anfangs dem neuen Investor sehr positiv gegenüber gestanden hätten. Diese bejahende Haltung, die die Managementliteratur auch als Phase des „Honeymoon“ des initialen und frühen Post Merger M\&A kennzeichnet (Cartwright und Cooper 2001, S. 77), begründet sich auch darin, dass sich die meisten Befragten als Geschäftsführer des bereits existierenden Unternehmens ihre neuen Eigner zuvor selbst mit ausgesucht oder sich selbst als neue Geschäftsführer in das M\&A eingekauft hatten. Die Manager betonten ihre kosmopolitische Offenheit. So beschrieb sich ein Manager als jemand, der ,wirklich in der Lage ist, andere Kulturen zu umarmen“. Gemeinhin verbreitete Ängste vor Akquisitionen, wie die Zerschlagung von funktionierenden Betrieben, Zusammenlegung oder Technologieabzug in Richtung Muttergesellschaften (vgl. Golinski und Henn 2015, S. 1, Franz et al. 2015, siehe Beitrag von Franz und Henn in diesem Heft), wurden explizit von den interviewten Managern für die Unternehmen, die sie leiten, zurückgewiesen.

Unterschiede zwischen eigenen Vorstellungen und dem wahrgenommenen Verhalten der neuen Eigner wurden für den weiteren Post Merger Prozess in allen Interviews formuliert, sei der neue Eigner ein westlicher Private EquityInvestor oder ein ausländischer Investor aus den Emerging Economies. Die Befragten hoben nahezu durchgängig hervor, sie seien nicht primär dem Finanzgeber verpflichtet, sondern ihrem Unternehmen mit seiner Belegschaft. Sie würden für ihre Entscheidungen „geradestehen“ und sich nicht hinter Entscheidungen des Management-Boards ,,verschanzen“. Ein Gesprächspartner in einem von einem Pri-
vate-Equity-Investor erworbenen Unternehmen erklärte die Herausforderungen an die von ihm verinnerlichte professionsethische Verantwortung: „,[Der Käufer] erzählt natürlich im Kaufprozess, dass er alles langfristig weiter entwickeln will, keinen sozialen Kahlschlag betreiben will; und die Realität kommt dann hinterher. So unter dem Motto: Der Wolf und die sieben Geißlein, ich bin die Großmutter und fresse die später. (...) Das ist ganz logisch, das machen alle. (...) Die Frage ist: Was ist dahinterliegend das strategische Eigeninteresse des Investors?"

Diese Verantwortungsethik geriet bei Private Equity-Investoren in keinen eklatanten Widerspruch während der weiteren Post Merger Entwicklung. Die Gesprächspartner betonten, die Zukunft sei weitgehend klar, der Wiederverkaufszeitpunkt des Unternehmens von Anfang an definiert, ebenso wie die Wege dorthin. Kennzahlensysteme, detailliertes Controlling etc. würden klare Maßstäbe liefern. Reorganisation gehöre zum Geschäft des neuen Eigners. Es wurde betont, dass der Private-Equity Investor strukturiert und nachvollziehbar arbeite. Zwar offenbarten sich Spannungen. Deutsche Führungskräfte beschrieben Private Equity-Investoren angesichts der hohen Wertschätzung von Kalkulierbarkeit und Modellierbarkeit oft als dem betrieblichen Alltag entrückt: „Deren Denkansatz ist: ,Wir können die Zukunft auf einem Excel Sheet modellieren' (...) So, dann steht dieses Excel Sheet und dann kommt die Realität und dann passt es auch irgendwann mal nicht mehr zusammen. (...) Da kommen natürlich auch zwei unterschiedliche Managementansätze aufeinander. Die Praktiker, die täglich (...) mit den Problemen umgehen - und dann die, die auf 30.000 Fuß einmal über Frankfurt geflogen sind und das Unternehmen für fünf, zehn Jahre modelliert haben." Doch bestand die Diskrepanz nicht darin, dass die Wahrnehmung des Gegenübers durch die deutschen Manager ihren eigenen professionsethischen Normen entgegenstehen würde, sondern vielmehr darin, dass genau diese professionsethischen Regeln von den neuen Eignern - aus Sicht der Interviewten - zu strikt angewendet wurden. In der Summe ergab sich so aus den vergleichsweise kleinen Differenzen zu den neuen Eignern eine Haltung der deutschen Manager, die mit Anpassungspragmatismus zu bezeichnen ist.

Ganz anders sahen Manager in Übernahmen aus China, Indien und Russland ihre professionsethischen Wertvorstellungen über Hierarchien und Prozesseffizienz durch die neuen Geschäftspartner infrage gestellt (Abb. 2). Im Falle chinesischer Übernahmen waren die untersuchten deutschen Unternehmen von großen chinesischen Staatskonglomeraten aufgekauft worden. Darin sahen sich die deutschen Manager oft auf einem verlorenen Außenposten. Abstimmungen mit der Zentrale in China bezeichneten sie als mühsam. In gemeinsamen Verhandlungen würde die formal-politische Position des Partners bedeutend mehr als dessen Sachkenntnis entscheiden. Hinzu kämen Probleme infolge 


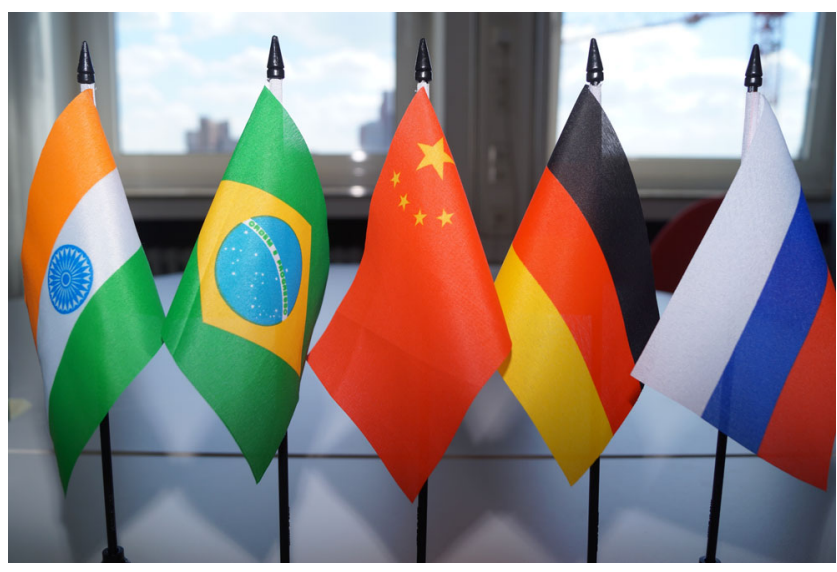

Abb. 2 BRIC-Investoren in Deutschland: Erst willkommen - die Probleme kommen später, Foto: M. Schalljo

mangelnder Englischkenntnisse der chinesischen Verhandlungspartner.

Insgesamt wurde auch die Zusammenarbeit mit den neuen Eignern aus Indien und Russland als aufwändig charakterisiert. Zwar seien die Gegenüber zumeist gut vorbereitet. Doch die Anfragen der neuen Muttergesellschaft würden nicht den gewohnten westlichen Benchmark-Schemata entsprechen, etwa wenn - anstelle aktueller und zukunftsorientierter Zahlen - Datenreihen bis weit in die Vergangenheit hinein abgefragt würden. Die Abläufe würden zudem gewohnte Routinen sprengen: „In Indien (...), die haben keine Prozesse. Doch, die haben einen Prozess, aber das Problem ist, die Leute kosten nichts. (...) Wo sie hier nur einen [Mitarbeiter] haben, haben sie da zwanzig Leute. (...) Anstatt jetzt Prozesse zu machen, effizient, setzen sie die Leute dahin. (...) Das heißt Planungen gibt es nicht.“ Und: „Die Jungs haben überhaupt keine Hemmungen aus einer halben Tagessitzung eine eineinhalb Tagessitzung werden zu lassen, um zum 37. Mal nachzufragen, warum dies so und so sein muss ...". Dies fordere den Manager als Teamleiter heraus, denn es hieße: ,den Atem anzuhalten, die Pferde zurückzuhalten, die Mitarbeiter trotzdem mitzuziehen“. Auch berichteten die deutschen Interviewpartner im Falle der Investoren aus China, Indien und Russland über die fehlende Absehbarkeit der Zukunft; es wäre völlig unklar, wohin die längerfristige Geschäftsstrategie verliefe.

Es könnte nun erwartet werden, dass die deutschen Führungskräfte folglich ihre eigenen Managementkompetenzen und -prinzipien den unerfahrenen, jungen Investoren vermitteln würden, kommen diese doch als Lernende bzw. „Knowledge Seekers“. Allerdings wird die Diskrepanz in der Expertise als zu groß angesehen, um Lernprozesse initiieren zu können: „Der Lernprozess ist nicht da, in keiner Weise." Die befragten Manager halten daher ihre internationalen Geschäftspartner so weit wie möglich aus dem laufenden Betrieb heraus. Ein Interviewpartner beschrieb sein
Gegenüber im Management-Board als „Fremdkörper“, ein anderer als „Strohmann“.

Die zentrale Bedeutung ihrer eigenen weitreichenden Handlungsautonomie begründeten die Manager damit, dass die Investoren aus den Emerging Economies ein funktionierendes Unternehmen mit fähigem Management gekauft hätten, und dieses zu erhalten sei im Interesse der Investoren selbst. Das hätte auch den Vorteil, dass dem Unternehmen nichts ,übergestülpt“" würde (siehe auch Franz und Henn in diesem Heft).

Wenn aber aus Sicht der Führungskraft der Investor nicht genug aus den Abläufen herausgehalten werden konnte, zeigten sich Spannungen. Befragt nach dem Einfluss des Investors auf das Leitbild des Unternehmens, antwortete ein Interviewpartner, er wolle angesichts hierarchischer Entscheidungsstrukturen, ineffizienter Abläufe und nicht nachvollziehbarer Forderungen das chinesische Gegenüber ,auch wirklich draußen halten aus dem Unternehmen. Also, wir selber geben das Leitbild nicht weiter. Wir hängen auch keine chinesische Fahne auf hier. Das mache ich auch nicht. Also, da müsste man mich schon rauswerfen, dass ich's tue. Und würde es dann auch nicht mehr machen, weil ich dann weg wär. (...) Mitarbeiter, die Öffentlichkeit, Kunden, alles was hier unser Umfeld ist, wäre irritiert und wäre nicht erfreut darüber (...).“ Er wies darauf hin, dass sein Team interkulturelle Trainings gemacht hätte, um die Führungskräfte in Umgangsformen einzuführen, wie etwa die Art Visitenkarten zu übergeben, mit Stäbchen zu essen und nicht den Anderen ins Gesicht „nein“ zu sagen. Diese Trainings ebenso wie seine eigene Selbstzuschreibung als kosmopolitisch offener Manager konnten aber die Logik, die diese massive Distanzierung begründete, nicht infrage stellen.

\section{Zusammenfassung und Ausblick}

Im Post Merger Prozess bewerteten die befragten deutschen Führungskräfte westliche Private Equity-Investoren als Chance zur eigenen Weiterentwicklung; die Befragten hatten lediglich Vorbehalte gegenüber einer zu starken Wertschätzung modellierter Wirklichkeit von Seiten der neuen Eigner, die die komplexe Alltagswelt von Führungskräften nicht berücksichtigen. Insgesamt lässt sich diese Positionierung mit einer Haltung des Anpassungspragmatismus beschreiben.

Dagegen zeigte sich generell eine Distanzierung zu den chinesischen, indischen und russischen Investoren. Damit gelang es den deutschen Managern, ihre Handlungsautonomie zu wahren und so die von ihnen verinnerlichten Regeln der westlichen Professionsethik in der gewohnten Weise zu realisieren. Dass es hier nicht zu einem wirklichen „Merger" - einer Verschmelzung - bzw. zu einer Annäherung 
mit weiteren positiven Synergieeffekten für beide Seiten kam (vgl. auch entsprechende Einsichten bei Si und Liefner 2014), ist ein bemerkenswertes Ergebnis angesichts dessen, dass große Diskrepanzen zwischen den Beteiligten oft als kritisch für den erfolgreichen Übernahmeprozess angesehen werden (Rottig et al. 2013). Zwar ist Distanz nicht immer schädlich für den Unternehmenserfolg (vgl. Ibert und Müller 2015); aber auf jeden Fall hat es Konsequenzen für die räumliche Organisation des M\&A, ob Investoren „nur“ als ferne Finanzgeber agieren, oder ob sich neue Potenziale für das deutsche Unternehmen beispielsweise durch Marktzugang auch in dem Land der jeweiligen Muttergesellschaft, mit den entsprechenden Wachstumsdynamiken der Emerging Economy, ergeben.

Die Studie arbeitet latente Deutungsmuster als verborgene, sozio-kulturell verankerte Steuerungskräfte in interkulturellen Zusammentreffen heraus. Eine professionsethisch begründete Distanzierung kann nicht durch kurze, oberflächliche interkulturelle Trainings überwunden werden; eine solche Annäherung würde nach Oevermann (1973/2001a, 2001b) eine „Krise“ erfordern, im Sinne der kritischen Notwendigkeit, sich - auf beiden Seiten intensiv und reflektierend mit den Routinen und der Tiefengrammatik der jeweils „Anderen“ auseinanderzusetzen.

Danksagung Wir danken der Deutschen Forschungsgemeinschaft (FU 424/15-1) für die Förderung des Projekts „,Deutungsmuster von Führungskräften bei Übernahmen und Beteiligungen durch ausländische Investoren“. Wir danken außerdem Ulrich Oevermann und seinem Frankfurter Team ebenso wie Irene Somm und der Kölner Interpretationsgruppe für die vielen Gespräche, die eine umfassende Interpretation unseres Interviewmaterials ermöglichten.

Open Access Dieser Artikel wird unter der Creative Commons Namensnennung 4.0 International Lizenz (http://creativecommons.org/ licenses/by/4.0/deed.de) veröffentlicht, welche die Nutzung, Vervielfältigung, Bearbeitung, Verbreitung und Wiedergabe in jeglichem Medium und Format erlaubt, sofern Sie den/die ursprünglichen Autor(en) und die Quelle ordnungsgemäß nennen, einen Link zur Creative Commons Lizenz beifügen und angeben, ob Änderungen vorgenommen wurden.

\section{Literatur}

Bathelt H, Gertler M (2005) The German variety of capitalism: forces and dynamics of evolutionary change. Econ Geogr 8(1):1-9. doi:10.1111/j.1944-8287.2005.tb00252.x
Bitterer N, Heeg S (2015) Die Macht der Zahlen. Kalkulative Praktiken in der Immobilienwirtschaft. Z Wirtschgeogr 59(1):34-50. doi: 10.1515/zfw.2015.0003

Brettel M, Kauffmann C, Kühn C, Sobczak C (2008) Private EquityInvestoren. Kohlhammer, Stuttgart

Cartwright S, Cooper C (2001) Managing mergers, acquisitions and strategic alliances: integrating people and cultures. Butterworth, Oxford

Franz M, Henn S, Weingarten J (2015) BRIC-Investitionen in Deutschland. Chancen und Risiken für Unternehmen und Arbeitnehmer. transcript, Bielefeld

Fuchs M, Schalljo S (2016) 'Western' professional ethics challenged by foreign acquisitions: German managers' patterns of interpretation surrounding Chinese and Indian investors. Geoforum 75(10):20-28. doi:10.1016/j.geoforum.2016.06.017

Goffman E (1974) Frame analysis: an essay on the organization of experience. Harper and Row, New York

Golinski S, Henn S (2015) Imperialisten, Spione oder Retter? Zur Charakterisierung von Direktinvestitionen aus Russland, Indien und China in deutschen Tageszeitungen. Z Wirtschgeogr 59(1):1-19. doi:10.1515/zfw.2015.0001

Ibert O, Müller F (2015) Network dynamics in constellations of cultural differences. Res Policy 44(1):181-194. doi:10.1016/j.respol. 2014.07.016

Oevermann U (2001a) Zur Analyse der Struktur von sozialen Deutungsmustern. Soz Sinn 2(1):3-33. doi:10.1515/sosi-2001-0102

Oevermann U (2001b) Die Struktur von sozialen Deutungsmustern Versuch einer Aktualisierung. Soz Sinn 2(1):35-81

Rottig D, Reus T, Tarba S (2013) The impact of culture on mergers and acquisitions. Adv Mergers Acquis 12:135-172. doi:10.1108/ s1479-361x(2013)0000012009

Schumpeter J (1928) Unternehmer. In: Handwörterbuch der Staatswissenschaften. Fischer, Jena, S 476-487

Si Y, Liefner I (2014) Cognitive distance and obstacles to subsidiary business success - the experience of Chinese companies in Germany. Tijdschr Econ Soc Geogr 105(3):285-300. doi:10.1111/ tesg. 12064

Weber M (1985) Wirtschaft und Gesellschaft. Mohr, Tübingen

Prof. Dr. Martina Fuchs Jg. 1962. Nach dem Studium in Trier und Göttingen 1989-1991 Promotion in Frankfurt, dann 1991-1993 Referentin bei der Volkswagen AG in Wolfsburg, 1993-1999 Habilitation in Düsseldorf. 2000-2002 Forschungsprojekte am Institut für Entwicklung und Frieden in Duisburg. 2002-2004 Professorin an der Universität Lüneburg, seit 2004 Professorin an der Universität zu Köln. Arbeitsschwerpunkte: Globalisierung und Lokalisierung von Unternehmen in Kernökonomien und Emerging Economies.

Dipl.-Geogr. Martin Schalljo Jg. 1983. Studium der Geographie an der Rheinischen Friedrich-Wilhelms-Universität Bonn (bis 2010). 2011-2013 freier Mitarbeiter bei einer mittelständischen Unternehmensberatung, Bonn. Seit 2013 wissenschaftlicher Mitarbeiter am Wirtschafts- und Sozialgeographischen Institut der Universität zu Köln. Arbeitsschwerpunkte: Ausländische Direktinvestitionen in der Bundesrepublik, theoretische Geographie, hermeneutische Methoden. 